\title{
A theorem on the absolute Cesàro summability of factored Fourier series.
}

\author{
By S. M. Mazhar (Univ. Allahabad, India)
}

Sammary. - In this paper, the author proves a theorem on the absolute CESARo summability of factored Founier series. His theorem extends a theorem of MATsumoto and genera. lizes a theorem of Prasad and BhatT.

1.1. Let $f(t)$ be a periodic function with period $2 \pi$ and integrable ( $L$. over $(-\pi, \pi)$ and let its FouRIER series be given by

$$
\begin{aligned}
f(t) & \sim \frac{1}{2} a_{0}+\sum_{1}^{\infty}\left(a_{n} \operatorname{Cos} n t+b_{n} \operatorname{Sin} n t\right) \\
& =\sum_{0}^{\infty} A_{n}(t) .
\end{aligned}
$$

We write

$$
\begin{array}{rlr}
\varphi(t) & =\frac{1}{2}\{f(x+t)+f(x-t)\} & \\
\Phi_{\alpha}(t) & =\frac{1}{\Gamma(\alpha)} \int_{0}^{t}(t-u)^{\alpha-3} \varphi(u) d u & \alpha>0, \\
\Phi_{0}(t) & =\varphi(t) &
\end{array}
$$

and

$$
\varphi_{\alpha}(t)=\Gamma(\alpha+1) t^{-\Phi_{\alpha}(t),} \quad \alpha \geq 0
$$

1.2. In 1948, OHewg proved the following theorem concerning the absolute summability factor of FoURIER series :

Theorem A. - If $\varphi_{\alpha}(t)(0 \leq \alpha \leq 1)$ is of bounded variation in $(0, \pi)$, then

$$
\Sigma A_{n}(t) /\{\log (n)\}^{1+\imath} \quad \in>0
$$

at the point $t=x$, is summable $|C, \alpha|$. 
Generalizing the above theorem of Cheng [1], Matsumoto [2] established the following theorem :

Theorem B. - If

$$
\int_{0}^{\pi}\left|d\left\{t^{-\Upsilon} \Phi_{\beta}(t)\right\}\right|<\infty
$$

then the series

$$
\sum_{0}^{\infty} n^{\gamma-\beta} A_{n}(t) /\{\log (n+2)\}^{1+z}
$$

at the point $t=x$, is summable $|O, \gamma|$, where $1 \geq \gamma \geq \beta \geq 0$ and $\varepsilon>0$.

The object of the present paper is to find in place of this special type of factor $1 /\{\log (n+2)\}^{1+\varepsilon}$ in the above theorem of Matsumoto [2], a general factor $\lambda_{n}$, where $\left\{\lambda_{n}\right\}$ is a convex sequence such that $\Sigma \lambda_{n} / n$ is convergent.

2.1. In what follows we shall prove the following theorem:

Theorem. - If $\left\{\lambda_{n}\right\}$ is a convex sequence such that $\Sigma \lambda_{n} / n<\infty$ and if

$$
\left.\int_{0}^{\pi}|d| t^{-\gamma} \Phi_{\beta}(t)\right\} \mid<\infty
$$

then the series

$$
\Sigma n^{\Upsilon-\beta} \lambda_{n} A_{n}(t)
$$

at the point $t=x$, is summable $|C, \gamma|$, where $1 \geq \gamma \geq \beta \geq 0$.

It may be remarked that this theorem also generalizes the following theorem of Prasad and BHatT [3].

THeOREM O. - If $\left\{\lambda_{n}\right\}$ is a convex sequence such that $\Sigma \lambda_{n} / n<\infty$ and $\varphi_{\alpha}(t)$ is of bounded variation in $(0, \pi)$, where $0 \leq \alpha \leq 1$, then the series $\sum_{n=0}^{\infty} \lambda_{n} A_{n}(t)$, at the point $t=x$, is summable $|C, \alpha|$.

2.2. The following lemmas will be required for the proof of our theorem :

Lfima 1. (Matsumoto [2]). - Let

$$
S_{k}(n, t)=\sum_{\nu=0}^{k} A_{n-\nu}^{r-1} \operatorname{Sin} \vee t, \quad k \leq n \text { and } 1 \geq \gamma>0
$$


S. M. Mazhar: A theorem on the absolute Cesar summability, etc.

13

then

$$
S_{k}(n, t)=\left\{\begin{array}{l}
O\left\{k(n-k) r^{-1}\right\} \\
O\left\{t^{-1}(n-k) r^{-1}\right\}
\end{array} \quad n>k\right.
$$

and

$$
S_{n}(n, t)=\left\{\begin{array}{l}
O(n r) \\
O(t-r)
\end{array}\right.
$$

Leman 2. (Matsumoto [2]). - Let

$$
S_{k}^{\lambda}(n, t)=\left(\frac{d}{d t}\right)^{\lambda} S_{k}(n, t) \quad \lambda \geq 1 \text { and } k \leq n,
$$

then we have

$$
S_{k}^{\lambda}(n, t)=\left\{\begin{array}{l}
O\left\{k^{\lambda+1}(n-k) r^{-1}\right\} \\
O\left\{k^{\lambda} t^{-1}(n-k) r^{-1}\right\}
\end{array} \quad k<n,\right.
$$

and

$$
S_{n}^{\lambda}(n, t)=\left\{\begin{array}{l}
O\left(n^{\lambda+r}\right) \\
O\left(n^{\lambda} t-r\right)
\end{array}\right.
$$

Lemma 3. - Let

$$
F(n, v)=(n-v)^{-1}\left|\Delta\left\{v^{\delta} \lambda_{\nu}\right\}\right|, \quad \delta=\gamma-\beta .
$$

If

$$
J(n, u)=\int_{u}^{\pi}(t-u)^{-\beta} \frac{d}{d t} H^{\Upsilon}(n, t) d t
$$

where

$$
H^{\Upsilon}(n, t)=\frac{1}{A_{n}^{\Upsilon}} \sum_{\nu=0}^{n} A_{n-\nu}^{\gamma-1} \gamma^{\delta} \lambda_{\nu} \operatorname{Sin} \nu t
$$

then

$$
J(n, u)=\left\{\begin{array}{l}
o\left[n^{\beta-\gamma}\left\{\sum_{v=0}^{n-1} v F(n, v)+n^{\gamma}+\delta \lambda_{n}\right\}\right] \\
o\left[n^{\beta-\gamma}\left\{\sum_{\nu=0}^{n-1} u^{-1} F(n, v)+n^{\delta} u^{-} \lambda_{n}\right\}\right] .
\end{array}\right.
$$


14

S. M. MazHaR: A theorem an the absolute desàro summability, etc.

Proof. - By partial summation we have

$$
\begin{aligned}
& H^{\gamma}(n, t)=\frac{1}{A_{\mu}^{\gamma}}\left\{\sum_{\nu=0}^{n=1} s_{\nu}(n, t) \Delta\left\{v^{\delta} \lambda_{\nu}\right\}+s_{n}(n, t) n^{\delta} \lambda_{n}\right\} \\
& =\left\{\begin{array}{l}
o\left[n-r\left\{\sum_{\nu=0}^{n-1} v F(n, v)+n \gamma+\delta \lambda_{n}\right\}\right] \\
o\left[n-r\left\{\sum_{\nu=0}^{n-1} t^{-1} F(n, v)+t-n^{\delta} \lambda_{n}\right\}\right]
\end{array}\right.
\end{aligned}
$$

by Lemma 1. Applying Lemma 2, we have

$$
\frac{d}{d t} H^{r}(n, t)=\left\{\begin{array}{l}
o\left[n-r\left\{\sum_{\nu=0}^{n-1} \nu^{2} F(n, \nu)+n^{\gamma+\delta+1} \lambda_{n}\right\}\right] \\
\left.o\left[n-\left.r\right|_{\nu=0} ^{n-1} v t^{-1} F(n, \nu)+n^{\delta+1} t-\gamma \lambda_{n}\right\}\right] .
\end{array}\right.
$$

Now

$$
\begin{aligned}
J(n, u) & =\left(\int_{u}^{u+n^{-1}}+\int_{u+n^{-1}}^{\pi}\right)(t-u)^{-\beta} \frac{d}{d t} H^{\gamma}(n, t) d t \\
& =I_{1}+I_{2}, \text { say. }
\end{aligned}
$$

It is easy to see that

$$
I_{1}=\left\{\begin{array}{l}
o\left[n^{\beta-1-\gamma}\left\{\sum_{\nu=0}^{n-1} v^{2} F(n, v)+n \gamma+\delta+1 \lambda_{n}\right\}\right] \\
o\left[n^{\beta-1-\gamma}\left\{\sum_{\nu=0}^{n-1} u^{-1} v F(n, v)+n^{\delta+1} u-\gamma \lambda_{n}\right\}\right],
\end{array}\right.
$$

and from second mean value theorem, we have

$$
\begin{aligned}
I_{2} & =n^{\beta} \int_{u+n^{-1}}^{\xi} \frac{d}{d t} H^{\gamma}(n, t) d t \\
& =\left\{\begin{array}{l}
0\left[n^{\beta-\gamma}\left\{\sum_{\nu=0}^{n-1} v F(n, \nu)+n^{\gamma+\delta} \lambda_{n}\right\}\right] \\
0\left[n^{\beta-\gamma}\left\{\sum_{\nu=0}^{n-1} u^{-1} F(n, \nu)+u-r n^{\delta} \lambda_{n}\right\}\right] .
\end{array}\right.
\end{aligned}
$$


LEMMA 4. - Let

$$
I(n, u)=\int_{0}^{u} v^{\Upsilon} \frac{d}{d v} J(n, v) d v
$$

then

$$
\left.I(n, u)=O\left[u^{\Upsilon} n^{\beta-\gamma} \mid \sum_{\nu=0}^{n-1} v F(n, v)+n^{\Upsilon}+\delta \lambda_{n}\right\}\right]
$$

Proof.

$$
\begin{array}{rlrl}
I(n, u) & =u^{\gamma} \int_{n}^{u} \frac{d}{d v} J(n, v) d v & 0<\eta<u \\
& =u^{\gamma}[J(n, v)]_{\eta}^{\mu} & \\
& =o\left[u^{\gamma} n^{\beta-\gamma}\left\{\sum_{\nu=0}^{n-1} v F(n, v)+n^{\gamma}+\delta \lambda_{n}\right\}\right]
\end{array}
$$

by using the first estimation for $J(n, u)$.

LFMMA 5. - Let

$$
K(n, u)=\int_{u}^{\pi} v^{\Upsilon} \frac{d}{d v} J(n, v) d v
$$

then, for $\beta>0$, we have

$$
\begin{aligned}
K(n, u)= & O\left[n^{\beta-Y}\left\{\sum_{\nu=0}^{n-1} u^{\Upsilon-1} F(n, v)+n^{\delta} \lambda_{n}\right\}\right] \\
& +o\left[n^{-\curlyvee}\left\{\sum_{\nu=0}^{n-1} u^{Y-\beta-1} F(n, v)+u^{-\beta} n^{\delta} \lambda_{n}\right\}\right] .
\end{aligned}
$$

Proof. - We have

$$
K(n, u)=\left[v^{\gamma} J(n, v)\right]_{u}^{\pi}-\gamma \int_{u}^{\pi} v^{\gamma-1} J(n, v) d v=K_{1}+K_{2}, \text { say. }
$$


Using second estimate of $J(n, x)$ of Lemma 3 , we have

$$
\begin{aligned}
K_{1} & =\pi^{\curlyvee} J(n, \pi)-u^{\Upsilon} J(n, u) \\
& =O\left[n^{\beta-\curlyvee}\left\{\sum_{\nu=0}^{n-1} u^{\gamma-1} F(n, \nu)+n^{\delta} \lambda_{n}\right\}\right] .
\end{aligned}
$$

Also

$$
\begin{aligned}
K_{2} & =-\gamma \int_{u}^{\pi} v^{\Upsilon-1} \int_{v}^{-\pi}(t-v)^{-\beta} \frac{d}{d t} H^{\Upsilon}(n, t) d t d v \\
& =-\gamma \int_{u}^{\pi} \frac{d}{d t} H^{\Upsilon}(n, t) \int_{u}^{t} v^{\gamma-1}(t-v)^{-\beta} d v d t \\
& =-\gamma \int_{u}^{\pi}\left(\frac{d}{d t}\right) H^{\Upsilon}(n, t) t^{\gamma-\beta} \int_{w / t}^{1} z^{\gamma-1}(1-z)^{-\beta} d z d t \\
& =-\gamma \int_{u \mid \pi}^{1} z^{\gamma-1}(1-z)^{-\beta} d z \int_{\xi}^{\pi} t^{\gamma-\beta} \frac{d}{d t} H^{\Upsilon}(n, t) d t \quad u<\xi<\pi, \\
& =O\left\{\int_{\xi}^{\pi} t^{\gamma-\beta} \frac{d}{d t} H^{\Upsilon}(n, t) d t\right\} .
\end{aligned}
$$

Substituting the second estimate of $H^{\Upsilon}(n, t)$ from (2.2.1), we have

$$
\begin{aligned}
K_{2} & =O\left\{\left[H^{\Upsilon}(n, t) t^{\gamma-\beta}\right]_{\xi}^{\pi}-(\gamma-\beta) \int_{\xi}^{\pi} H^{\Upsilon}(n, t) t^{\gamma-\beta-1} d t\right\} \\
& \left.=O\left[n-\gamma \int_{\nu=0}^{n-1} u^{\gamma-\beta-1} F(n, \nu)+u^{-\beta} n^{\delta} \lambda_{n}\right\}\right] .
\end{aligned}
$$

This completes the proof of Lemma 5.

3.1. Proof of the theorem. - Case $(i): \gamma>\beta>0$.

It is sufficient to show that

$$
\sum_{1}^{\infty}\left|\zeta_{n}^{Y}\right| / n<\infty
$$


where $\zeta_{n}$ is the $n$-th CESÀro mean of order $\gamma$ of the sequence $\left\{n^{1+\delta} \lambda_{n} A_{n}(x)\right\}$ i. $\theta$.

$$
\zeta_{n}^{r}=\frac{1}{A_{n}^{r}} \sum_{\nu=0}^{n} A_{n \rightarrow \nu}^{r-1} \vee \gamma^{\delta} \lambda_{\nu} A_{\nu}(x) \quad \delta=\gamma-\beta
$$$$
=\frac{1}{A_{n}^{\gamma}} \sum_{\nu=0}^{n} A_{n-y}^{\gamma-1} v v^{\delta} \lambda_{y} \frac{2}{\pi} \int_{0}^{\pi} \varphi(t) \operatorname{Cos} v t d t
$$$$
=\frac{2}{\pi} \int_{0}^{\pi} \varphi(t) \frac{d}{d t}\left\{\frac{1}{A_{n}^{\gamma}} \sum_{\nu=0}^{n} A_{n-\nu}^{\gamma-1} \nu^{\delta} \lambda_{\nu} \operatorname{Sin} \nu t\right\} d t
$$$$
=\frac{2}{\pi} \int_{0}^{\pi} \varphi(t) \frac{d}{d t} H^{\Upsilon}(n, t) d t
$$$$
=\frac{2}{\pi} \int_{0}^{\pi} \frac{d}{d t} H^{\Upsilon}(n, t) \frac{1}{\Gamma(1-\beta)} \int_{0}^{t}(t-u)^{-\beta} d \Phi_{\beta}(u)
$$$$
=\frac{2}{\pi \Gamma(1-\beta)} \int_{0}^{\pi} d \Phi_{\beta}(u) \int_{u}^{\pi}(t-u)^{-\beta} \frac{d}{d t} H^{Y}(n, t) d t
$$$$
=\frac{2}{\pi \Gamma(1-\beta)} \int_{0}^{\pi} d \Phi_{\mathbf{\beta}}(u) J(n, u)
$$$$
=-\frac{2}{\pi \Gamma(1-\beta)} \int_{0}^{\pi} \Phi_{\beta}(u) \frac{d}{d u} J(n, u) d u
$$

$$
\begin{aligned}
=- & \frac{2}{\pi \Gamma(1-\beta)}\left[u-\Upsilon \Phi_{\beta}(u) I(n, u)\right]_{0}^{\pi}+ \\
& \frac{2}{\pi \Gamma(1-\beta)} \int_{0}^{\pi} I(n, u) d\left\{\Phi_{\beta}(u) u-\Upsilon\right\} .
\end{aligned}
$$

Thus, it is sufficient to prove that

$$
\sum_{1}^{\infty} \frac{|I(n, \pi)|}{n}<\infty
$$


and

$$
\sum_{1}^{\infty} \frac{|I(n, u)|}{n}<\infty
$$

uniformly with respect to $u$, for $0<u<\pi$.

Proof of (3.1.3). - Taking $\varphi(t)=t^{\varphi-\beta}$, we have easily

$$
\Phi_{\beta}(t)=\frac{\Gamma(\gamma-\beta+1) t^{\gamma}}{\Gamma(\gamma+1)}
$$

therefore,

$$
d\left\{u^{-} \Phi_{\beta}(u)\right\}=0
$$

Also

$$
\begin{aligned}
\int_{0}^{\pi} \varphi(t) \operatorname{Cos} v t d t & =\int_{0}^{\pi} t^{\gamma-\beta} \operatorname{Cos} v t d t \\
& \simeq \nu^{-1-\gamma+\beta} \Gamma(\gamma-\beta+1) \operatorname{Cos} \frac{\pi}{2}(\gamma-\beta+1) \quad \text { (TITCHMARSH [4]). }
\end{aligned}
$$

Hence from (3.1.1) and (3.1.2), we have

$$
I(n, \pi)==0\left\{\frac{1}{A_{n}^{r}} \sum_{\nu=0}^{n} A_{n \rightarrow-y}^{\gamma-1} \lambda_{y}\right\}
$$

so that

$$
\begin{aligned}
\Sigma \frac{|I(n, \pi)|}{n} & =O\left\{\sum_{1}^{\infty} n^{-r-1} n^{\varphi-1}\right\}+O\left\{\sum_{1}^{\infty} n^{-Y-1} \sum_{1}^{n} A_{n-\nu}^{r-1} \lambda_{\nu}\right\} \\
& =O(1)+O\left\{\sum_{1}^{\infty} \lambda_{\nu} \sum_{\nu}^{\infty}(n-v+1)^{Y-1} n^{-Y-1}\right\} \\
& =O(1)+O\left\{\sum_{1}^{\infty} \lambda_{\nu} \int_{\nu}^{\infty}(x-v)^{\varphi-1} x^{-r-1} d x\right\} \\
& =O(1)+O\left\{\sum_{1}^{\infty} \frac{\lambda_{v}}{v}\right\}=O(1) .
\end{aligned}
$$


S. M. MazHaR: A theorem on the absolute Cesàro summability, etc.

19

Proof of (3.1.4). - Let

$$
\sum_{n=1}^{\infty} \frac{1}{n}|I(n, u)|=\sum_{\Sigma}^{\left[\frac{1}{u}\right]}+\underset{\sum}{\sum_{\left[\frac{1}{u}\right]+1}^{\infty}}=M_{1}+M_{2} \text {, say. }
$$

Applying Lemma 4, we find that

$$
\begin{aligned}
& M_{1}=0\left[\left[\frac{\left[\frac{1}{u}\right]}{\Sigma} u_{1}^{\gamma} n^{\beta-\gamma-1}\left\{\left\{_{0}^{n-1} \vee E(n, v)+n^{\gamma}+\delta \lambda_{n}\right\}\right]\right.\right. \\
& =O\left[\sum_{2}^{\left[\frac{1}{u}\right]} u^{Y} n^{\beta-Y-1} \sum_{\nu=0}^{n-1} v(n-v)^{Y-1} \mid \Delta\left\{v^{\delta} \lambda_{y}\right\}\right]+O\left[\sum_{2}^{\left[\frac{1}{u}\right]} u^{Y} n^{Y-1} \lambda_{n}\right] \\
& =M_{11}+M_{12} \text {, say. }
\end{aligned}
$$

Now

$$
M_{12}=O\left(u^{r}\left[\frac{1}{u}\right]^{\varphi}\right)=O(1)
$$

and

$$
\begin{aligned}
& M_{11}=O\left\{u^{\gamma} \sum_{1}^{\left[\frac{1}{v}\right]} n^{\beta-\gamma-\tau} \sum_{1}^{n}(n-\nu+1)^{\gamma-1 /(\nu-1)}\left|\Delta\left\{(\nu-1)^{\delta} \lambda_{\nu-1}\right\}\right|\right\} \\
& =O\left\{u r \frac{\left[\frac{1}{u}\right]}{\sum}(\nu-1)\left|\Delta\left\{(\nu-1)^{\delta} \lambda_{\nu-1}\right\}\right|\right. \\
& \left.\times \sum_{n=\nu}^{\left[\frac{1}{w}\right]} n^{\beta-\gamma-1}(n-\nu+1)^{r-1}\right\} \\
& =0\left\{u^{\gamma} \sum_{1}^{\left[\frac{k}{u}\right]}(\nu-1)\left|\Delta\left\{(\nu-1)^{\delta} \lambda_{y-1}\right\}\right| v^{\beta-1}\right\} \\
& \left.=0, u^{\gamma} \sum_{1}^{\left[\frac{1}{w}\right]}(\nu-1)^{\delta+1} \Delta \lambda_{\nu-1} \nu \beta-1\right\} \\
& +o\left\{u^{r} \sum_{\Sigma}^{\left[\frac{1}{u}\right]} v^{\delta} \lambda_{\nu} v^{B-1}\right\} \\
& =M_{111}+M_{112} \text {, say. }
\end{aligned}
$$




$$
M_{11 L}=O\left\{u^{r}\left[\frac{1}{u}\right]^{r} \sum_{1}^{\left[\frac{1}{2}\right]} \Delta \lambda_{y \rightarrow 1}\right\}=O(1), \text { since } \Delta \lambda_{y} \geq 0
$$

and

$$
\left.\left.M_{112}=0 \nmid u^{r}\left[\frac{1}{u}\right]^{\gamma} \sum_{1}^{\left[\frac{1}{u}\right.}\right]_{v}{\frac{\lambda_{y}}{v}}_{v}\right\}=O(1)
$$

Therefore $M_{1}=O(1)$.

As regards $M_{2}$, we have

$$
I(n, u)=I(n, \pi)-k(n, u)
$$

and since we have already proved in (3.1.5) that

$$
\sum_{n=1}^{\infty} \frac{|I(n, \pi)|}{n}<\infty
$$

it is sufficient to prove that

$$
\sum_{n=\left[\frac{1}{u}\right]+1}^{\infty} \frac{|k(n, u)|}{n}=O(1)
$$

uniformly in $u$, for $0<u<\pi$.

Now

$$
\begin{aligned}
\sum_{n=\left[\frac{1}{u}\right]+1}^{\infty} \frac{|k(n, u)|}{n}=0\left\{\sum_{n=\left[\frac{1}{u}\right]+1}^{\infty}\left[u^{Y-1} n^{\beta-Y-1^{n}-\sum_{\nu=0}^{-1}}(n-v)^{Y-1}\left|\Delta\left\{v^{\delta} \lambda_{\nu}\right\}\right|\right]\right. \\
\left.+\frac{\lambda_{n}}{n}+n^{-1-\beta} u^{-\beta} \lambda_{n}\right\} \\
=M_{21}+M_{22}+M_{23}, \text { say. }
\end{aligned}
$$


It is obvious that $M_{22}$ and $M_{23}$ are bounded and

$$
\begin{aligned}
& M_{21}=0\left\{u^{\gamma-1} \sum_{n=\left[\frac{1}{v}\right]+1}^{\infty} n^{\beta-\gamma-1} \sum_{\nu=0}^{n-1}(n-v)^{\gamma-1}\left|\Delta\left\{v^{\delta} \lambda_{\nu}\right\}\right|\right\} \\
& =O\left\{u^{\gamma-1} \underset{\nu=0}{\left[\frac{1}{w}\right]}\left|\Delta\left(v^{\delta} \lambda \nu\right)\right| \underset{n=\left[\frac{1}{u}\right]+1}{\stackrel{\infty}{y}} n^{\beta-\gamma-1}(n-v)^{\gamma-1}\right\} \\
& +o\left\{u^{\gamma-1} \sum_{\nu=\left[\frac{1}{z}\right]}^{\infty}\left|\Delta\left(v^{\delta} \lambda \nu\right)\right| \sum_{\nu+1}^{\infty} n^{\beta-\gamma-1}(n-v)^{\gamma-1}\right\} \\
& =M_{211}+M_{212} \text {, say. }
\end{aligned}
$$

Now

$$
\begin{aligned}
M_{212} & =0\left\{\begin{array}{l}
u^{\gamma-1} \\
\sum_{\nu=\left[\frac{1}{u}\right]}^{\infty}\left(v^{\delta} \Delta \lambda_{\nu}+v^{\delta-1} \lambda_{\nu+1}\right) v^{\beta-1}
\end{array}\right. \\
& =O\left\{u^{\gamma-1}\left[\frac{1}{u}\right]^{\gamma-1}\right\}=O(1), \text { as } u \rightarrow 0 .
\end{aligned}
$$

Since, in the case of $M_{211}, v<\left\lfloor\frac{1}{u}\right\rfloor+1$, we have

$$
\begin{aligned}
M_{211}= & \left.0\left\{u^{\gamma-1} \sum_{n=\left[\frac{1}{u}\right]+1}^{\infty} n^{\beta-2}\right\}+0\right\} u^{\gamma-1} \sum_{1}^{\left.\frac{1}{u}\right]}\left|\Delta\left\{v^{\delta} \lambda_{\nu}\right\}\right| \\
& \left.\times \int_{\left[\frac{1}{u}\right]+1}^{\infty} x^{\beta-\gamma-1}\left(x-\left[\frac{1}{u}\right]-1\right)^{\gamma-1} d x\right\} \\
= & O\left\{u^{\gamma-1}\left[\frac{1}{u}\right]^{\beta-1}\right\}+0\left\{u^{\gamma-1}\left[\frac{1}{u}\right]^{\beta-1} \sum_{\frac{1}{u}}^{\left[\frac{1}{u}\right]}\left(v^{\delta} \Delta \lambda_{\nu}+v^{\delta-1} \lambda_{\nu+1}\right)\right\} \\
= & O\left\{u^{\gamma-1}\left[\frac{1}{u}\right]^{\gamma-1}\right\}=O(1) .
\end{aligned}
$$

Hence

$$
M_{2}=O(1)
$$

This proves the theorem for $\beta>0$. 
Case (ii): When $\gamma>\beta=0$. In this case, we are given that

$$
\int_{0}^{\pi} \mid d\left\{t^{-\gamma} \varphi^{\prime}(t)||<\infty, \quad 0<y \leq 1\right.
$$

and we have to show that $\sum_{0}^{\infty} n^{\gamma} \lambda_{n} A_{n}(x)$ is summable $|C, \gamma|$, that is, we have to establish the convergence of the series $\Sigma\left|\zeta_{n}\right| / n$, where $\left({ }^{1}\right)$

$$
\begin{aligned}
& \zeta_{n}^{\Upsilon}=\frac{1}{A_{n}^{\Upsilon}} \sum_{\nu=0}^{n} A_{n-1}^{Y-1} \nu \nu^{Y} \lambda_{y} A_{\nu}(x) \\
& =\frac{2}{\pi} \int_{0}^{\pi} \varphi(t) \frac{d}{d t} H^{\Upsilon}(n . t) d t \\
& =\frac{2}{\pi} \int_{0}^{\pi}\left(t^{-\gamma} \varphi(t)\right)\left(t^{r} \frac{d}{d t} H^{\Upsilon}(n, t)\right) d t \\
& =0\left\{\int_{0}^{\pi} v^{\gamma} \frac{d}{d v} H^{\Upsilon}(n, v) d v\right\} \\
& +o\left\{\int_{0}^{\pi} d\{t-\varphi(t)\} \int_{0}^{t} v^{\varphi} \frac{d}{d v} H^{\Upsilon}(n, v) d v\right\} .
\end{aligned}
$$

Writing

$$
\bar{I}(n, t)=\int_{0}^{t} v^{\Upsilon} \frac{d}{d v} H^{\Upsilon}(n, v) d v,
$$

it is sufficient to show that

$$
\sum_{i}^{\infty} \frac{|\vec{I}(n, t)|}{n}<\infty
$$

uniformly in $t$, for $0<t \leq \pi$. Patting $\varphi(t)=t^{r}$, we have, as in the case $(i)$

$$
\bar{I}(n, \pi)=O\left\{\frac{1}{A_{n}^{\gamma}} \sum_{\nu=0}^{n} A_{n-\nu}^{\Upsilon-1} \lambda_{\nu}\right\} .
$$

(i) From this place onward we shall be using the same notations as in the case $(i)$ but with the obvious change that here $\delta=\gamma$. 
Hence

$$
\sum_{1}^{\infty} \frac{|\bar{I}(n, \pi)|}{n}=O\left\{\sum_{1}^{\infty} n^{-\gamma-1} \sum_{\nu=0}^{n}(n-\nu+1)^{r-1} \lambda_{\nu}\right\}=O(1) \text {, as shown }
$$

in (3.1.5). It, therefore, remains to show that

$$
\underset{\mathbf{1}}{\infty} \frac{|\vec{I}(n, t)|}{n}<\infty
$$

uniformly in $t$ for $0<t<\pi$.

Now

$$
\sum_{1}^{\infty} \frac{|\bar{I}(n, t)|}{n}=\frac{\left[\begin{array}{l}
1 \\
t
\end{array}\right]}{\Sigma}+\sum_{1}^{\infty}=N_{1}+N_{2}, \text { say. }
$$

By the second mean value theorem and the first estimation of (2.2.1) we have

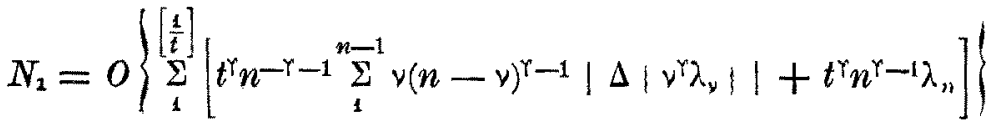

$$
\begin{aligned}
& =0\left\{t^{r} \sum_{i}^{\left[\frac{1}{t}\right]} v|\Delta| v^{r} \lambda_{y}|| \sum_{\nu+1}^{\left[\frac{1}{t}\right]} n^{-r-1}(n-v)^{\gamma-1}\right\}+O(1) \\
& =O\left\{t^{r} \sum_{1}^{\left[\frac{1}{t}\right]}|\Delta| r^{r} \lambda_{\nu}||\right\}+O(1)=O(1) .
\end{aligned}
$$

Also

$$
N_{3}=O\left\{\sum_{n=\left[\frac{1}{t}\right]+1}^{\infty}\left\lfloor\frac{|\bar{I}(n, \pi)|}{n}+\frac{|\bar{K}(n, t)|]}{n}\right]\right\}
$$

where

$$
\bar{K}(n, t)=\int_{t}^{\pi} v^{r} \frac{d}{d v} H^{\Upsilon}(n, v) d v
$$


Now

$$
\sum_{n=\left[\frac{1}{t}\right]+1}^{\infty} \frac{|\bar{I}(n, \pi)|}{n}<\infty
$$

and from the second relation for $H^{Y}(n, v)$, we bave

$$
\begin{aligned}
& \bar{K}(n, t)=\left[v^{\gamma} H_{-}^{\Upsilon}(n, v)\right]_{t}^{\pi}-\gamma \int_{t}^{\pi} v^{\gamma-1} H^{\Upsilon}(n, v) d v \\
& =0\left\{n^{-Y} t^{Y-1}{ }_{y=0}^{n-1}(n-y)^{y-1}|\Delta| v^{Y} \lambda_{y}||+\lambda_{n}\right\} \\
& -\frac{\gamma}{A_{n}^{Y}} \sum_{\nu=0}^{n} A_{n-y}^{\gamma-1} \nu^{\gamma} \lambda_{\nu} \int_{t}^{\pi} v^{\gamma-1} \operatorname{Sin} v v d v \\
& =X_{1}+X_{2} \text {, say. }
\end{aligned}
$$

But

$$
\begin{aligned}
\sum_{n=\left[\frac{1}{t}\right]+1}^{\infty} \frac{\left|X_{1}\right|}{n}=0 & \left\{\sum_{n=\left[\frac{1}{t}\right]+1}^{\infty} \frac{\lambda_{n}}{n}\right\}+0 \mid \sum_{n=\left[\frac{1}{t}\right]+1}^{\infty} t^{\gamma-1} n^{-Y-1} \\
& \left.\times \sum_{\nu=0}^{n-1}(n-v)^{\gamma-1}|\Delta| v^{\gamma} \lambda_{\nu}||\right\} \\
= & O(1),
\end{aligned}
$$

by proceeding as in the proof of the relation:

$$
M_{21}=O(1)
$$


Also

$$
\begin{aligned}
& \sum_{n=\left[\frac{1}{t}\right]+1}^{\infty} \frac{\left|X_{2}\right|}{n}=0\left\{\sum_{n=\left[\frac{1}{t}\right]+1}^{\infty} n^{-r-1} \sum_{\nu=1}^{n} A_{n-\nu}^{\gamma-1} \nu^{r-1} \lambda_{\nu} t^{\gamma-1}\right\} \\
& =O\left\{t^{r-1} \sum_{\nu=1}^{\left[\frac{1}{t}\right]+1} v^{\gamma-1} \lambda_{y} \sum_{n=\left[\frac{1}{t}\right]+1}^{\infty} n^{-r-1} A_{n-\nu}^{r-1}\right\} \\
& +o\left\{t^{\gamma-1} \sum_{\nu=\left[\frac{1}{t}\right]+1}^{\infty} v^{\gamma-1} \lambda_{y} \sum_{n=\nu}^{\infty} n^{-\gamma-1} A_{n-\nu}^{\gamma-1}\right\} \\
& =O\left\{t^{\gamma-1}\left[\frac{1}{t}\right]^{\gamma-1}\left[\sum_{\nu=1}^{\left[\frac{1}{t}\right]+1} \frac{\lambda_{\nu}}{v}\right\}+0\left\{t^{\gamma-1} \sum_{\nu=\left[\frac{1}{t}\right]+1}^{\infty} v^{\gamma-2 \lambda_{\nu}}\right\}\right. \\
& =O\left\{t^{\gamma-1}\left[\frac{1}{t}\right]^{\gamma-1}\right\}=O(1) .
\end{aligned}
$$

This completes the proof of the theorem for the case $\gamma>\beta=0$.

Case (iii): When $\gamma=\beta>0$. The proof of the theorem for this case is similar to that of Case $(i)$ and hence we omit it

Case $(i v)$ : When $\gamma=\beta=0$. In this case $A_{n}(x)=O\left(\frac{1}{n}\right)$, hence

$$
\Sigma \lambda_{n}\left|A_{n}(x)\right|=\Sigma O\left\{\frac{\lambda_{n}}{n}\right\}<\infty \text {. }
$$

This completes the proof of the theorem.

The anthor is much indebted to Prof. B. N. Prasad, F. N. I, for his kind encouragement and helpful guidance.

\section{REFERENCES}

[1] M. T. Cheng, Summability factors of Fourier series at a given point, \& Duke Mathematical Journal », 15 (1948), pp. 29.36.

[2] K. Matsumoto, On absolute Cesàto summability of a series related to a Fourier series, "Tôhoku Mathematical Journal ", 8 (1956), pp. 205.222.

[3] B. N. Prasad and S. N. BHATt, The summability factors of a Fourier series, "Duke Mathematical Journal :, 24 (1957), pp. 103-117.

[4] E. C. Titomanase, The Theory of Functions, (1952), Oxford. 